\title{
Exacerbated Insomnia
}

National Cancer Institute

\section{Source}

National Cancer Institute. Exacerbated Insomnia. NCI Thesaurus. Code C35609.

Insomnia that is made worse by other factors, including pain, physiological or psychological disorders, external causes, or pharmacologic interventions. 